\title{
ABSOLUTE BAIRE SETS
}

\section{STELIOS NEGREPONTIS}

1. Introduction. Definitions and facts. For background in notation and terminology the reader should consult [2]. We make here the blanket assumption that all spaces to be considered in this note are completely regular Hausdorff topological spaces.

A continuous map $f: X \rightarrow Y$ is called proper if $f$ is onto, closed, continuous, and if for every compact set $K$ in $Y, f^{-1}(K)$ is compact.

The family of Baire sets of a space $X$ is the $\sigma$-field generated by the zero-sets of $X$. The family of Borel sets of a space $X$ is the $\sigma$-field generated by the closed sets of $X$. For a metric space the two families coincide, but in general the family of Borel sets properly contains the family of Baire sets.

A subset $A$ of a space $X$ is said to be $C^{*}$-embedded in $X$ if every real-valued continuous bounded function on $A$ is the restriction of a real-valued continuous (bounded) function on $X$. Thus, for example, a space $X$ is $C^{*}$-embedded in its Stone-Cech compactification $\beta X$.

2. Absolute Baire spaces. We will now prove our first main result, which characterizes the spaces which are Baire sets in their StoneCech compactifications. The characterization, which uses the idea included in the proof of Theorem D, $\$ 51$, of Halmos's text on measure theory [2], is surprisingly simple.

2.1. ThEOREM. The following are equivalent on any space $X$ :

(1) $X$ is Baire in $\beta X$ (i.e. $X$ is an "absolute Baire set").

(2) There is a separable metric space $\Gamma$ which is an absolute Borel space (in the class of separable metric spaces) and a proper mapping $\phi: X \rightarrow \Gamma$.

Proof. $(1) \Rightarrow(2)$. Suppose that $X$ is a Baire set in $\beta X$. There is a sequence of zero-sets of $\beta X$, say $Z_{n}, n=1,2, \cdots$, such that $X$ belongs to the $\sigma$-field generated by $\left\{Z_{n}, n=1,2, \ldots\right\}$. We choose $f_{n} \in C(\beta X)$ such that $0 \leqq f_{n} \leqq 1$ and $Z_{n}=Z\left(f_{n}\right)$, and we define $d(x, y)$ $=\sum_{n=1}^{\infty}\left(1 / 2^{n}\right)\left|f_{n}(x)-f_{n}(y)\right|$. Clearly $d$ is a continuous pseudometric on $\beta X$. Let $\Xi$ be the metric space associated with the pseudometric space $(\beta X, d)$. The identity mapping $\pi: \beta X \rightarrow \Xi$ is continuous and onto, and, therefore, $\Xi$ is a compact (metric) space. Arguing as in Halmos, we conclude that there is a subset $\Gamma_{n}$ of $\Xi$ such that $\pi^{-1} \Gamma_{n}$ 1966.

Presented to the Society, January 25, 1966; received by the editors February 19, 
$=Z_{n}$ for $n=1,2, \cdots$. This clearly implies that there is some $\Gamma$ in the $\sigma$-field generated by $\left\{\Gamma_{n}, n=1,2, \cdots\right\}$ such that $\pi^{-1} \Gamma=X$. Now $\pi \pi^{-1} \Gamma_{n}=\Gamma_{n}=\pi Z_{n}$; hence, $\Gamma_{n}$ is compact, and thus a Borel set of $\Xi$; hence $\Gamma$ is also a Borel set of $\Xi$. Thus, $\Gamma$ is a separable metric space which is an absolute Borel space in the class of separable metric spaces. It is easily seen that the mapping $\pi: X \rightarrow \Gamma$ is proper.

$(2) \Rightarrow(1)$. Suppose that there is a proper mapping $\pi: X \rightarrow \Gamma$, where $\Gamma$ is an absolute Borel separable metric space. Let $\Delta$ be a compact metric space which is a compactification of $\Gamma$. (Such a space $\Delta$ exists; e.g. we embed $\Gamma$ in a countable product of unit intervals and let $\Delta$ be its closure.) Let $\tilde{\pi}: \beta X \rightarrow \Delta$ be the Stone-Cech extension of $\pi$. By [4, Lemma 1.5], $\bar{\pi}(\beta X-X)=\Delta-\Gamma$ and, hence, $\tilde{\pi}^{-1}(\Gamma)=X$. Thus, $X$ is a Baire set in $\beta X$. (This is almost immediate because the inverse image under a continuous mapping of a zero set is again a zero set; and $\Gamma$, being an absolute Borel set, is a Borel subset of $\Delta$.)

2.2. REMARK. The author has not been able to prove thus far that the following conditions (3), (4) are also equivalent to (1):

(3) $X$ is a Baire set in some compactification of $X$,

(4) $X$ is a Baire set in every compactification of $X$. Of course, it is true that $(4) \Rightarrow(3) \Rightarrow(1)$.

2.3. REMARK. We could also define Baire sets of class $\alpha$ and relate them to the separable metric absolute Borel spaces of class $\alpha$; such a classification will not be undertaken here.

2.4. Corollary. If $X$ is a Baire set in $\beta X$, then $X$ is a Lindelöf space.

Proof. Use Theorem 2.1 and [4, Theorem 2.2].

2.5. Corollary. A metric space which is a Baire set in $\beta X$ is separable.

2.6. Theorem. Let $A$ be a Baire set in a compact space. Then $A$ is a Baire set in $\beta X$; in particular, every Baire set in a compact space is Lindelöf.

Proof. The proof is similar to that of the first half of Theorem 2.1, and it is omitted.

The proof of the following lemma is omitted.

2.7. Lemma. Let $A \subset B \subset C$ be inclusions of spaces, with $B C^{*}$ embedded in $C$. If $A$ is a Baire set in $B$, and $B$ is a Baire set in $C$, then $A$ is a Baire set in $C$. 
2.8. Theorem. Let $X$ be an absolute Baire set. Then every Baire set of $X$ is an absolute Baire set.

Proof. A Baire set of $X$ is, by 2.7, a Baire set of $\beta X$. Hence, by 2.6, it is an absolute Baire set.

2.9. Lemma. Let $X_{n}$ be a sequence of absolute Baire sets. Then the product $X=\Pi_{n} X_{n}$ is also an absolute Baire set.

Proof. We know that $X_{n}$ is a Baire set in $\beta X_{n}$. Let $B X=\Pi_{n} \beta X_{n}$; clearly, $X=\bigcap_{n} \Pi_{n}^{-1}\left(X_{n}\right)$, and, thus, $X$ is easily seen to be a Baire set in $\beta X$; hence, $X$ is a Baire set in $\beta X$.

2.10. Theorem. A countable product of absolute Baire spaces is an absolute Baire space, and, thus, Lindelöf.

2.11. Corollary. The product of a sequence of locally compact, $\sigma$-compact spaces is Lindelöf.

2.12. Corollary. If $X$ is an absolute Baire space, then $\beta X-X$ is Lindelöf.

2.13. Example. A $\sigma$-compact space which is not an absolute Baire space.

Let $N$ be the set of positive integers, and let $p \in \beta N-N$. The set $\Sigma=N \cup(p)$ is countable and, hence, $\sigma$-compact; in fact, every point is a $G_{\delta}$. But $\beta \Sigma-\Sigma=\beta N-N-(p)$ is easily seen to be a pseudocompact space, which is not compact, and, hence, certainly not a Baire set in $\beta N$. By $2.12, \Sigma$ is not an absolute Baire space.

3. Baire sets in $\beta X-X$.

3.1. The main objective in this paragraph is to show that if $X$ is locally compact, $\sigma$-compact, then every Baire set of $\beta X-X$ is $C^{*}$. embedded. There is some background to this theorem. Gillman and Henriksen [1] have proved that if $X$ is as above, then every complement of a zero-set in $\beta X-X$ is $C^{*}$-embedded. Since a complement of a zero-set is certainly a Baire set, the following theorem includes this result.

3.2. THEOREM. If $X$ is a locally compact, $\sigma$-compact space, then every Baire set of $\beta X-X$ is $C^{*}$-embedded in $\beta X$.

Proof. Let $A$ be a Baire set in $\beta X-X$. Notice that the fact that $X$ is a locally compact, $\sigma$-compact space implies that $\beta X-X$ is a zero-set in $\beta X$, and, hence, a $C^{*}$-embedded Baire set in $\beta X$. There- 
fore, by 2.7, $A$ is a Baire set in $\beta X$. Hence, the set $A \cup X$ is also a Baire set in $\beta X$; thus, by 2.6, $A \cup X$ is a Lindelöf space, and in particular, a normal space. Notice further that $A$ is closed in the relative topology of $A \cup X$, and, hence, it is $C^{*}$-embedded in $A \cup X$. Further, since $X \subset A \cup X \subset \beta X$, we see that $A \cup X$ is $C^{*}$-embedded in $\beta X$. By transitivity, $A$ is $C^{*}$-embedded in $\beta X$, and a fortiori, in $\beta X-X$.

3.3. REMARK. It is instructive to see how we can prove the Gillman-Henriksen theorem mentioned above by a direct argument. In fact, let $A$ be a cozero-set of $\beta X-X$; since $\beta X-X$ is compact, $A$ is $\sigma$-compact, and, hence, $A \cup X$ is also $\sigma$-compact, and, therefore, normal. The rest of the argument proceeds as above. ${ }^{1}$

\section{REFERENCES}

1. L. Gillman and M. Henriksen, Rings of continuous functions in which every finitely generated ideal is principal, Trans. Amer. Math. Soc. 82 (1956), 366-391.

2. L. Gillman and M. Jerison, Rings of continuous functions, Van Nostrand, Princeton, N. J., 1960.

3. P. R. Halmos, Measure theory, Van Nostrand, New York, 1950.

4. M. Henriksen and J. R. Isbell, Some properties of compactifications, Duke Math. J. 25 (1958), 83-105.

INDIANA UNIVERSITY

1 Note: It has come to the attention of the author, after the completion of this paper, that Z. Frolik, in his paper On coanalytic and bianalytic spaces, Bull. Acad. Polon. Sci. 12 (1964), 527-530, has obtained a different proof of Theorem 2.1. 